\title{
Upregulation of NEK2 is associated with drug resistance in ovarian cancer
}

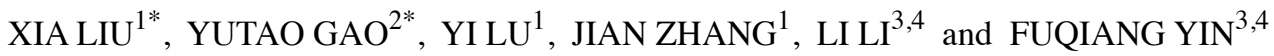 \\ ${ }^{1}$ Center for Translational Medicine, Guangxi Medical University, Nanning, Guangxi 530021; ${ }^{2}$ Department of \\ Obstetrics and Gynecology, Beijing Chao-Yang Hospital, Affiliated to Capital Medical University, Chaoyang, \\ Beijing 100020; ${ }^{3}$ Medical Scientific Research Centre, Guangxi Medical University, Nanning, Guangxi 530021; \\ ${ }^{4}$ Key Laboratory for High-Incidence-Tumor Prevention and Treatment, Ministry of Education, P.R. China
}

Received October 23, 2013; Accepted November 22, 2013

DOI: $10.3892 /$ or.2013.2910

\begin{abstract}
NEK2 [NIMA (never in mitosis gene A)-related expressed kinase 2] is associated with various biological behaviors in the development of cancer, while research concerning its association with drug resistance is limited. The association of NEK2 with drug resistance in ovarian cancer has not yet been reported. In the present study, on the basis of microarray results from Oncomine and the GEO Profiles online database, we revealed that NEK2 mRNA expression in ovarian cancer tissues is upregulated. In addition, its expression in drugresistant ovarian cancer cells was upregulated when compared with expression with their sensitive or parental counterparts. Finally, we performed a comprehensive bioinformatic analysis consisting of protein/gene-protein/gene interaction network, annotation of biological processes and microRNA-mRNA interaction analysis. We observed that NEK2 directly or indirectly interacts with a number of genes, proteins, microRNAs and biological processes associated with drug resistance in ovarian and other types of cancer. These results indicate that NEK2 contributes to drug resistance in ovarian cancer and it may be an important therapeutic target.
\end{abstract}

\section{Introduction}

Ovarian cancer is the leading cause of death among malignancies of the female reproductive system, with a high rate of mortality worldwide. Early-stage ovarian cancer is frequently asymptomatic and difficult to detect; thus, most patients are in advanced stages [International Federation of Gynecology and Obstetrics (FIGO) stage III and IV] at the time of initial diag-

Correspondence to: Dr Fuqiang Yin, Medical Scientific Research Centre, Guangxi Medical University, 22 Shuangyong Road, Nanning, Guangxi 530021, P.R. China

E-mail: yinfq@mail2.sysu.edu.cn

*Contributed equally

Key words: NEK2, drug resistance, ovarian cancer, bioinformatics nosis (1), and 5-year survival rates are less than $40 \%$ with only modestly improved survival noted over the past 40 years (2). The current therapy for ovarian cancer is debulking surgery followed by cisplatin-centered chemotherapy (3). Although cisplatin-centered chemotherapy achieves a complete response rate of 40 to $60 \%$ in advanced ovarian cancer patients (4), long-term survival remains poor as a result of recurrence and emergence of drug resistance that finally leads to fatal disease (5).

Drug resistance, both intrinsic and acquired, results from a variety of factors including individual variations in patients and somatic cell genetic differences in tumors (6). A number of factors such as decreased cell-associated drugs, altered drug inactivation, increased DNA damage tolerance/repair, increased anti-apoptotic regulator activity and growth factor receptor deregulation are considered to be responsible for drug resistance in ovarian cancer $(7,8)$. However, regardless of the mechanisms, abnormal expression of drug resistance-related genes often plays an important role in drug resistance. And among all of these drug resistance-related genes, oncogenes are clearly the key players.

Oncogenes refer to genes whose activation can contribute to the development of cancer (9) and many are involved in drug resistance in varied types of cancers. For example, the overexpression and activation of the $c-m y c$ oncogene is associated with drug resistance in small cell lung carcinoma (10); the $L R F$ oncogene is a survival factor in chondrosarcoma and contributes to tumor malignancy and drug resistance (11) and the STAT3 oncogene is a predictive marker of drug resistance in cancer (12). In ovarian cancer, a total of 13 oncogenes including CCNE1, PIK3CA, RAB25, MYC, PRKC1, FGF1, NOTCH3, PIK3R1, AKT2, EGFR, ERBB2, KRAS and AURKA are associated with cancer development (13), and several genes such as KRAS (14), ERBB2 (15), PIK3CA $(16,17)$ and $P I K 3 R I$ (18) are involved in drug resistance.

NEK2 [NIMA (never in mitosis gene A)-related expressed kinase 2], a serine/threonine centrosomal kinase, is highly expressed and activated during the $\mathrm{S}$ and $\mathrm{G} 2$ phases of the cell cycle (19). NEK2 has emerged as an important oncogene due to its regulatory role in mitosis (20). NEK2 has been proven to play pivotal roles in the development of several types of cancers, while its relationship with ovarian cancer is rare. 
More recently, NEK2 has become important since two studies indicate that high expression of NEK2 induces drug resistance in multiple myeloma $(21,22)$. However, despite these study, the research on NEK2 in regards to drug resistance in cancers is still limited, and its relationship with drug resistance in ovarian cancer has never been reported. In the present study, on the basis of a comprehensive bioinformatic analysis, for the first time, we report that NEK2 may contribute to drug resistance in ovarian cancer.

\section{Materials and methods}

The target gene, NEK2, which is closely associated with drug resistance in multiple myeloma $(21,22)$ was selected for bioinformatic analysis.

Databases. The microarray data of NEK2 in ovarian cancer tissues was retrieved from the Oncomine online database (https://www.oncomine.org/resource/main.html); the microarray data of NEK2 in ovarian cancer cells was retrieved from the Gene Expression Omnibus (GEO) (http://www.ncbi. nlm.nih.gov/geoprofiles/) (23). The protein/gene-protein/gene interaction network was generated using the GeneMANIA online tool (http://www.genemania.org/) (24); annotation of biological processes was performed using the COREMINE online tool (http://www.coremine.com/medical/) (25). The microRNAs (miRNAs) targeted to the gene were predicted by miRWalk online tool which included 7 prediction tools (DIANAmT, miRanda, miRDB, miRWalk, RNAhybrid, PICTAR5, Targetscan) (http://www.umm.uni-heidelberg. de/apps/zmf/mirwalk/) (26).

NEK2 expression in ovarian cancer cells retrieved from the GEO database was normalized. Unpaired, two-tailed t-test assuming homogeneity of the variances was performed with Excel software.

\section{Results}

Function of NEK2 in cancers. Genetic screening for cell division cycle mutants in the filamentous fungus Aspergillus nidulans resulted in the discovery of never in mitosis A (NIMA), a serine/threonine kinase that is essential for mitotic entry. Since then, NIMA-related kinases (NEKs) have been identified in most eukaryotes, including humans in which 11 genetically distinct proteins named NEK1 to NEK11 have been discovered (27). The NEKs play crucial roles in several aspects of mitotic progression, such as chromatin condensation, nuclear envelope breakdown, spindle assembly checkpoint signaling and cytokinesis. Of the human NEKs, NEK 2 is the most closely related to Aspergillus NIMA and is the first NEK which has been relatively well studied (27). Similar to NIMA, NEK2 is a cell cycle regulated kinase, with a peak of expression and activity in the $\mathrm{S}$ and $\mathrm{G} 2$ phase of the cell cycle, and it is a core component of the human centrosome $(28,29)$. NEK2 also contributes to the establishment of the microtubule-based mitotic spindle (27).

It has been proven that the NEK2 protein is elevated 2- to 5-fold in cell lines derived from a wide range of human tumors including those of cervical, ovarian, breast, prostate and leukemic origin (30), suggesting its potential roles in cancer development. The role of NEK2 in breast cancer has been extensively studied. In various human breast cancer cell lines, suppression of NEK2 was found to induce aneuploidy and cell cycle arrest resulting in cell death. Significantly, the breast cancer cell line which was most sensitive to NEK2 depletion was of the triple negative breast cancer subtype. These results indicate that NEK2 plays crucial roles in breast cancer growth at primary and secondary sites (31). Similarly, Wang et al (32) indicated that the abnormal expression of NEK 2 and $\beta$-catenin may be one of the mechanisms for tumorigenesis, particularly for abnormal tumor proliferation, and the cytoplasmic expression of NEK2 is associated with both tumor grade and tumor size. These results suggest that NEK 2 and $\beta$-catenin may represent new potential targets for therapeutic intervention. Moreover, a study indicated that the genetic polymorphisms of NEK2 are related to breast cancer susceptibility in Chinese Han women (33). In addition, the $\mathrm{NEK} 2 \mathrm{C}$, which is a splice variant of $\mathrm{NEK} 2$, which was found to have significantly upregulated expression in breast cancer cell lines as well as in human primary breast cancer tissue, plays a crucial role in breast cancer development and NEK2C inhibition may be a useful therapeutic target for human breast tumors (34). In addition to the important roles in breast cancer, NEK 2 also plays a role in other types of cancer. For instance, NEK2 is shown to be involved in the development of lung cancer induced by smoking and affects patient survival (35). In cervical cancer, NEK2 was induced in 102 cancer biopsies when compared with 24 normal controls, indicating its potential roles in cervical cancer (36).

The role of NEK2 in ovarian cancer is less understood. Limited studies indicate that NEK2 is overexpressed in ovarian cancer cells (30) and its expression is regulated by NR2F2 which is associated with a significantly shorter disease-free interval (37), yet no further research has been reported. Likewise, the relationship between NEK2 and drug resistance is unclear, with only several studies indicating its drug resistance-related functions. The combined use of both NEK2 siRNA and cisplatin inhibited cell proliferation and induced apoptotic cell death in vitro, suggesting that NEK 2 may be associated with drug resistance in colorectal cancer (38). Similarly, the combination of NEK2 siRNA with paclitaxel and doxorubicin was found to improve the sensitivity of breast cancer cells during chemotherapy treatment (20). More recently, two studies strongly support the idea that NEK2 contributes to drug resistance in cancers. Zhou et al (21) and Harrison (22) revealed that high expression of NEK2 induced drug resistance mainly through activation of efflux pumps and it may be a strong predictor of drug resistance and poor prognosis in multiple myeloma and other types of cancers.

Taken together, NEK2, an oncogene, plays crucial roles in the development of various types of cancers, but related studies in ovarian cancer are rare. In addition, although several studies have revealed the drug resistance-related functions of NEK 2 in several cancers, studies in this area are limited and its association with drug resistance in ovarian cancer has never been reported.

Expression of NEK2 in ovarian cancer and drug-resistant cells. NEK 2 is considered as an oncogene and is overexpressed in various tumors. Thus, as an oncogene, NEK 2 should be 

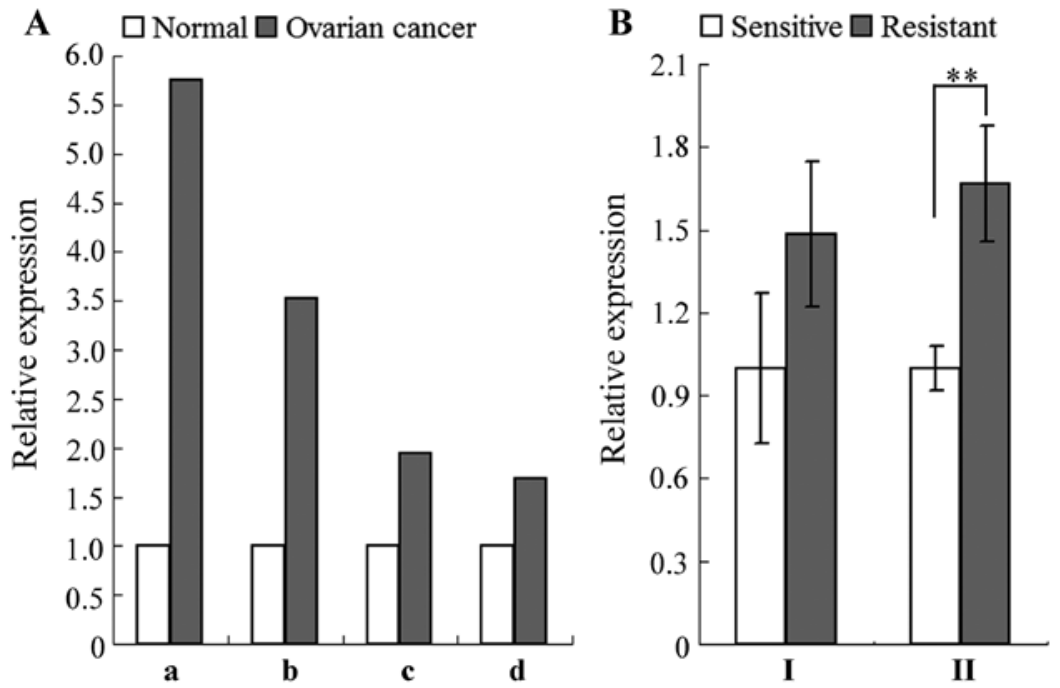

Figure 1. Relative expression of NEK2 in ovarian cancer. (A) Relative expression of NEK2 in ovarian cancer tissues and normal control tissues based on the array data retrieved from the Oncomine online database. (a) The TCGA Ovarian Statistics which covered 8 cases of normal controls and 509 ovarian cancer tissues ( $\mathrm{p}=8.51 \mathrm{e}-7)$. (b) The Bonome Ovarian Statistics which covered 10 cases of normal controls and 185 ovarian cancer tissues ( $\mathrm{p}=2.75 \mathrm{e}-10)$. (c) The Welsh Ovarian Statistics which covered 4 cases of normal controls and 28 ovarian cancer tissues (p=2.37e-6). (d) The Hendrix Ovarian Statistics which covered 4 cases of normal controls and 37 ovarian cancer tissues ( $\mathrm{p}=4.82 \mathrm{e}-4)$. All these data in the Oncomine database are presented as fold changes (ovarian cancer vs. normal). In the present study, the expression of NEK2 in the normal control was normalized to 1.0, and all data are presented as relative expression. (B) The relative expression of NEK2 in cisplatin-sensitive and -resistant ovarian cancer cells based on the array data retrieved from the GEO Profiles database. (I) Cisplatin-sensitive and -resistant A2780 ovarian cancer cells (GDS3754/211080_s_at/NEK2), with 5 biological replications each (ID: 66834845). (II) Cisplatin-sensitive and -resistant A2780 ovarian cancer cells (GDS3754/204641_at/NEK2), with 5 biological replications each (ID: 66828489). All data in the GEO Profiles database are presented as expression values. In the present study, the expression of NEK2 in sensitive cancer cells was normalized to 1.0 , and all data are presented as relative expression. ${ }^{* *}$ Significant difference in statistics. NEK2, NIMA (never in mitosis gene A)-related expressed kinase 2; GEO, Gene Expression Omnibus.

overexpressed in ovarian cancer when compared to the normal control and should be upregulated in drug-resistant cells when compared to sensitive cells. Based on microarray data retrieved from the Oncomine online database, we revealed that among 4 independent microarray analyses which covered a total of 759 ovarian cancer tissues and 26 normal controls, NEK 2 was overexpressed 1.687- to 5.754-fold in ovarian cancer tissues when compared with the normal controls (Fig. 1A). Regarding two microarray data sets from the GEO Profiles database, NEK2 was found to be elevated 1.5- to 1.7-fold in cisplatinresistant A2780 ovarian cancer cells when compared with the sensitive counterparts (Fig. 1B). These results suggest that NEK2 may be involved in the development of ovarian cancer, including the regulation of drug resistance.

Functional prediction and analysis based on protein/ gene-protein/gene interaction. The protein/gene interaction of NEK2 with other proteins/genes was analyzed using GeneMANIA online tool. As shown in Fig. 2, NEK2 has direct interactions with 8 proteins/genes. Among these, NEK2 was co-expressed with RAD51, BRCA2, PTEN and DAPK1, was co-expressed and had genetic interaction with BRCA1, was co-expressed and shared protein domains with CDK2, shared protein domains with CHEK2 and had genetic interactions with NOM1. With the exception of NOM1, the other 7 proteins/genes are all associated with drug resistance in ovarian cancer.

BRCA1 and BRCA2 proteins are critically important for the repair of double-strand breaks (DSB) by homologous recombination (HR) (39). They cooperate in DNA damage responses in a PALB2-dependent manner and have important implications for the genesis of ovarian cancer and for chemotherapy with DNA interstrand cross-linking agents (40). The mRNA expression of BRCA1 has a negative correlation with the clinical sensitivity of platinum-based chemotherapy (41). In addition, BRCA1 is positively correlated with MDR1 which is significantly involved in drug resistance and disease progression (42). In regards to BRCA2, previous research indicates that the functional restoration of BRCA2 due to a secondary BRCA2 mutation is involved in acquired drug resistance of BRCA2-mutated ovarian carcinomas (43). These results implicate the important role of BRCA1 and BRCA2 in drug resistance in ovarian cancer. $\operatorname{Rad} 51$ functionally interacts with BRCA1 in the meiotic and mitotic cell cycles (44), and, on the basis of an interaction network (Fig. 2), Rad51 co-expressed, physically interacted and shared pathways with BRCA1, and co-expressed, and shared pathways with BRCA2. These results indicate that Rad51 which is closely associated with BRCA1 and BRCA2 may also have drug resistance-related functions in ovarian cancer. The PTEN tumor-suppressor is a central negative regulator of the PI3K/AKT signaling cascade and suppresses cell survival as well as cell proliferation. It is found to be either inactivated or mutated in various human malignancies. Previously studies suggest that PTEN may be involved in drug resistance via the PI3K/AKT pathway and the p53-mediated apoptotic cascade. Reduction in PTEN expression was found to result in the development of drug resistance in OVCAR-3 cells and the alterations conferred resistance to cisplatin through the activation of PI3K/Akt and the inhibition of Bax translocation (17). Further research indicates that overexpression of PTEN reverses chemoresistance to cisplatin in human ovarian cancer cells through inactivation of the 
Table I. Annotated functions of NEK2 with its interacting proteins/genes based on the protein/gene-protein/gene interaction network.

\begin{tabular}{lll}
\hline Annotated function & FDR $^{\mathrm{a}}$ & NEK2 and other proteins/genes in the network \\
\hline $\begin{array}{l}\text { Cell cycle-related } \\
\text { Regulation of mitotic cell cycle }\end{array}$ & $2.08 \mathrm{e}-7$ & $\begin{array}{l}\text { NEK2, BRCA2, TP53, TP63, TP73, CDK2, APC, } \\
\text { PTEN, CDKN1A, CDKN1B }\end{array}$ \\
$\begin{array}{l}\text { Centrosome cycle } \\
\text { G2/M transition of mitotic cell cycle }\end{array}$ & $2.02 \mathrm{e}-4$ & NEK2, BRCA1, BRCA2, CDK2 \\
Regulation of mitosis & $1.99 \mathrm{e}-2$ & NEK2, CHEK2, CDK2, CDKN1A \\
Centrosome organization & $5.76 \mathrm{e}-2$ & NEK2, PTEN, APC \\
Microtubule-related & $8.97 \mathrm{e}-4$ & NEK2, BRCA1, BRCA2, CDK2 \\
Microtubule cytoskeleton organization & & \\
Microtubule organizing center organization & $3.66 \mathrm{e}-4$ & NEK2, BRCA1, BRCA2, CDK2, CHEK2, APC \\
Microtubule-based process & $1.18 \mathrm{e}-3$ & NEK2, BRCA1, BRCA2, CDK2 \\
Regulation of microtubule cytoskeleton organization & $1.47 \mathrm{e}-3$ & NEK2, BRCA1, BRCA2, CDK2, CHEK2, APC \\
Regulation of microtubule-based process & $3.07 \mathrm{e}-2$ & NEK2, BRCA1, APC \\
\end{tabular}

${ }^{a}$ FDR, false discovery rate; NEK2, NIMA (never in mitosis gene A)-related expressed kinase 2.

PI3K/AKT cell survival pathway and may serve as a potential molecular target for the treatment of chemoresistant ovarian cancer (45). Moreover, overexpression of PTEN upregulates p53 content and increases the sensitivity of chemoresistant cells to cisplatin-induced apoptosis without detectable changes in the levels of phosphorylated Akt, suggesting that PTEN may be involved in drug resistance through a p53-mediated apoptotic cascade independent of the PI3K/Akt pathway (46). CHEK2 is one of the critical kinases governing cell apoptosis, cell cycle checkpoint and DNA damage repair. In ovarian cancer cells, CHEK2 is degraded at the protein level in response to cisplatin through the ubiquitin-proteasome pathway, suggesting that degradation or decreased expression of CHEK 2 is partially responsible for chemoresistance (47). The expression of DAPK1 and DAPK2 is altered in multi-drug-resistant gastric cancer cell lines and thus these genes may be an integral part of the mechanisms responsible for chemoresistance (48). In ovarian cancer, DAPK1 has direct interactions with FBXO32, PDCD4, PTEN, TP53 and TP73, and has indirect interactions with BRCA1, BRCA2, CDKN1A, IL24, MLH1 and SULF1, which are all involved in drug resistance in ovarian cancer, suggesting its potential role in drug resistance in ovarian cancer (49). As for CDK2, a previous study revealed that cisplatin consistently induces transient $\mathrm{S}$-phase arrest by inhibiting the CDK2/cyclin A complex in the S-phase at $12 \mathrm{~h}$ after treatment with cisplatin or DAP in combination with the mitotic inhibitor nocodazole and also potently inhibits G1-phase CDK2/cyclin E activities at $18 \mathrm{~h}$ (50), indicating that CDK 2 can directly respond to cisplatin in ovarian cancer cells.

In addition to those with direct interactions with NEK2, there were other proteins/genes in the network which indirectly interacted with NEK2 (Fig. 2). Among those, MLH1 (51,52), PDCD4 (53), TP53 (54), TP73 (55), WWOX (56), CDKN1A (57), PLAGL1, PYCARD, APC and TP63 (49), PALB2 (40), ABCC3 (58) and PMS2 (59) are associated with drug resistance in ovarian cancer.
Collectively, based on the protein/gene interaction network analysis, 8 proteins/genes were identified which directly interact with NEK2, and 7 of them were found to contribute to drug resistance in ovarian cancer; 24 proteins/genes were found to indirectly interact with NEK2 and 13 of them were found to be associated with drug resistance in ovarian cancer. These results indicate a potential role of NEK2 in drug resistance in ovarian cancer.

Functional annotation of NEK2 with its interacting proteins/genes was performed to further reveal the relationship of NEK2 with drug resistance. As shown in Table I, the main functions of NEK2 with its interacting proteins/genes are cell cycle-related and microtubule-related functions, which were both proven to be associated with drug resistance in ovarian and other cancer types. Cell cycle-mediated drug resistance is best described as a relative insensitivity to a chemotherapeutic agent because of the position of the cells in the cell cycle. The cell cycle is closely involved in the chemosensitivity to combination chemotherapy, and the chemotherapeutic agents correlated with cell cycle events include taxanes, platinum, camptothecin and fluorouracil (60). It has been proven that the cell cycle is closely associated with drug resistance in ovarian cancer. Integration of DNA methylation and gene expression reveals specific platinum resistance-related signaling pathways in ovarian cancer, which include cell growth-promoting pathways, PI3K/Akt and cell cycle progression (61). Moreover, numerous genes participate in drug resistance through regulation of the cell cycle. For example, Phb1 induces G0/G1 phase cell cycle arrest and promotes cancer cell survival, and silencing of Phb1 expression is a valuable therapeutic approach for chemoresistant ovarian cancer by increasing the sensitivity of cancer cells to apoptosis (62). Likewise, comprehensive bioinformatic analysis revealed that 15 tumor-suppressor genes (TSGs) associated with drug resistance in ovarian cancer perform their drug resistance-related functions through 5 pathways including 


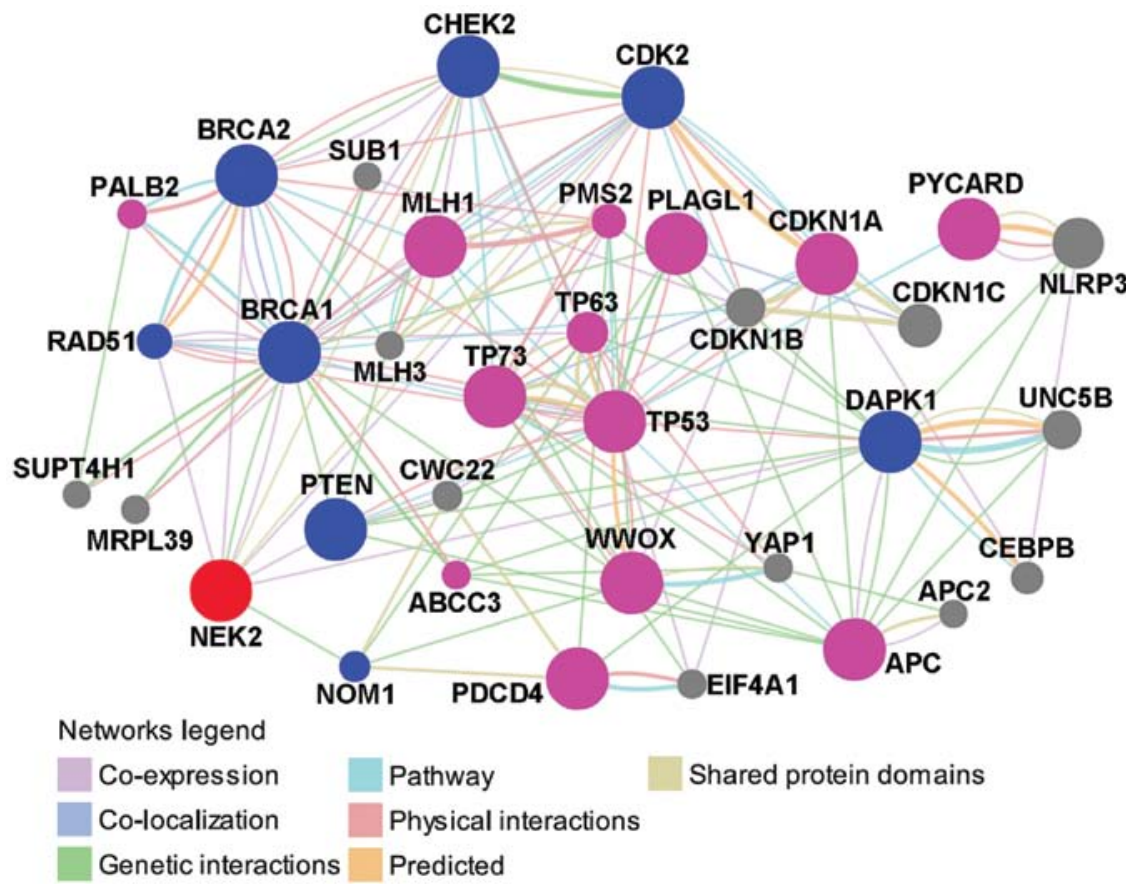

Figure 2. Protein/gene-protein/gene interaction network of NEK2 generated using the GeneMANIA online tool. The query in blue includes the proteins/genes which directly interact with NEK2; the query in pink includes the drug resistant-related proteins/genes which indirectly interact with NEK2. The type of interactions between proteins/genes are illustrated as indicated by the network legend. NEK2, NIMA (never in mitosis gene A)-related expressed kinase 2.

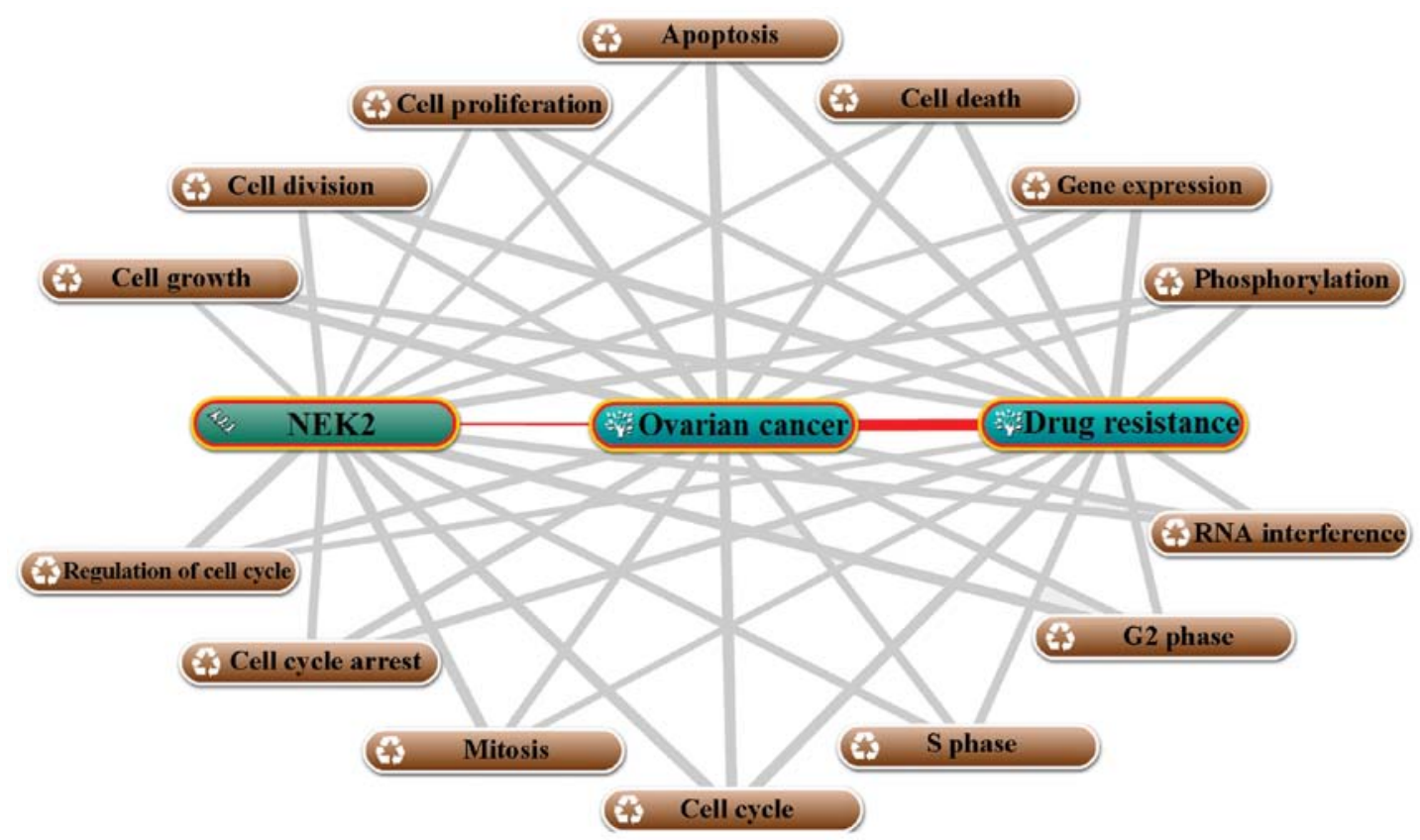

Figure 3. Annotation of the biological processes of NEK2 with ovarian cancer and drug resistance using the COREMINE online database/tool. The top 14 biological processes $(\mathrm{p}<0.00265)$ which were closely related to the three terms are annotated. NEK2, NIMA (never in mitosis gene A)-related expressed kinase 2.

the cell cycle (49). In addition, the cell cycle is also involved in drug transportation in cancer. Multidrug resistance driven by $\mathrm{ABC}$ membrane transporters is one of the major reasons for treatment failure in human malignancies, and modulation of MDR by cell cycle-related factors has been observed in MCF-7 breast cancer cells (63).

Microtubules are intracellular tubular structures found in all eukaryotic cells. Microtubules have various functions including organization of intracellular structure, cell division and intracellular transport (64). However, abnormal changes in microtubules lead to drug resistance in cancers. For instance, RASSF1A, tumor-suppressor protein, forms an endogenous complex with tubulin and promotes the stabilization of microtubules. Previous research revealed a strong correlation between the reduced relative expression of RASSF1A and taxol resistance in primary ovarian cancer. The reason is that 
Table II. The top 10 miRNAs targeted by NEK2 as predicted by miRNA-mRNA interactions and their drug resistance-related functions in cancer.

\section{microRNA-mRNA prediction tool}

\begin{tabular}{|c|c|c|c|c|c|c|c|c|}
\hline \multirow[b]{2}{*}{ microRNA } & & \multirow{2}{*}{$\begin{array}{l}\text { Drug resistance-related functions } \\
\text { of the microRNAs in cancers (ref.) }\end{array}$} \\
\hline & a & $\mathrm{b}$ & $\mathrm{c}$ & $\mathrm{d}$ & $\mathrm{e}$ & $\mathrm{f}$ & $\mathrm{g}$ & \\
\hline hsa-miR-27a & 1 & 1 & 0 & 1 & 1 & 1 & 1 & Drug resistance in ovarian cancer (67) \\
\hline hsa-miR-27b & 1 & 1 & 0 & 1 & 1 & 1 & 1 & Drug resistance in Ehrlich ascites tumor cells (68) \\
\hline hsa-miR-150 & 1 & 1 & 1 & 1 & 0 & 1 & 1 & Drug resistance and metastasis in ovarian cancer (69) \\
\hline hsa-miR-374a & 1 & 1 & 1 & 1 & 0 & 1 & 1 & Drug resistance in head and neck squamous cell carcinoma cells (70) \\
\hline hsa-miR-374b & 1 & 1 & 1 & 1 & 0 & 1 & 1 & No reported functions in cancer \\
\hline hsa-miR-630 & 1 & 1 & 1 & 1 & 0 & 1 & 1 & Drug resistance in non-small cell lung cancer (71) \\
\hline hsa-miR-24-2 & 1 & 1 & 0 & 1 & 0 & 1 & 1 & Drug resistance in human cancer cells (MCF-7 and HeLa) (72) \\
\hline hsa-miR-216b & 1 & 1 & 0 & 1 & 0 & 1 & 1 & $\begin{array}{l}\text { Cell proliferation, invasion and tumor growth and cellular senescence } \\
\text { in nasopharyngeal carcinoma and colorectal cancer }(74,75)\end{array}$ \\
\hline hsa-miR-128 & 0 & 1 & 1 & 1 & 0 & 1 & 1 & Drug resistance in breast cancer (73) \\
\hline hsa-miR-486-5p & 1 & 1 & 0 & 1 & 0 & 1 & 1 & Cell migration and invasion in non-small cell lung cancer (76) \\
\hline
\end{tabular}

The miRNA-mRNA analysis tools: a, DIANAmT; b, miRanda; c, miRDB; d, miRWalk; e, RNAhybrid; f, PICTAR5; g, Targetscan; NEK2, NIMA (never in mitosis gene A)-related expressed kinase 2.

the loss of RASSF1A expression sensitizes cells to microtubule destabilizing stimuli finally resulting in the development of drug resistance (65). In ovarian cancer treatment, anticancer drugs including paclitaxel, epothilone B (EpoB) and discodermolide all specifically interfere with microtubules and arrest cells in the G2/M phase of the cell cycle (66).

Collectively, on the basis of functional annotation, the two ovarian cancer drug resistance-related functions, including cell cycle- and microtubule-related functions, were annotated to be the major functions of NEK2 with its interacting proteins/genes of which most are associated with drug resistance in ovarian cancer (Fig. 2). These results suggest that NEK2 may contribute to drug resistance in a cell cycle- and/or microtubule-mediated manner in ovarian cancer.

Functional prediction and analysis based on annotation of biological processes. The biological process annotation was performed using COREMINE online database/tool. As shown in Fig. 3, a total of 14 biological processes were annotated with NEK2, ovarian cancer and drug resistance $(\mathrm{p}<0.00265)$, which suggested that, on the one hand, those biological processes contribute to the development of ovarian cancer and drug resistance, and on the other hand, NEK2 was associated with these biological processes and it may be a regulator for those processes. Taken together, the annotation of biological processes suggested that NEK2 may contribute to drug resistance via 14 biological processes in ovarian cancer. The 14 biological processes may be mainly divided into two groups including growth/death-related processes which include cell growth, cell division, cell proliferation, apoptosis and cell death and cell cycle-related processes including cell cycle arrest, regulation of cell cycle, mitosis, the cell cycle, $\mathrm{S}$ and G2 phase. These results indicate that NEK2 may participate in the regulation of ovarian cancer drug resistance mainly through the cell cycle, cell growth and death, in particular, through the cell cycle. This conclusion was consistent with the result of our functional annotation (Table I).

Functional prediction and analysis based on miRNA-mRNA interaction. As shown in Table II, the top 10 miRNAs targeted to NEK 2 were predicted by 7 prediction tools, and the drug resistance-related functions of these miRNAs were reviewed and integrated. Among the top $10 \mathrm{miRNAs}, 7$ are associated with drug resistance in ovarian and other cancers. For instance, the expression levels of miR-27a were found to be increased in paclitaxel-resistant ovarian cancer cell line A2780/taxol when compared with its parental line A2780. Transfection of A2780/ taxol cells with inhibitors of miR-27a enhanced the sensitivity of A2780/taxol cells to paclitaxel and increased paclitaxelinduced apoptosis, which suggest that the deregulation of miR-27a may be involved in the development of drug resistance in ovarian cancer (67). miRNA expression profiles revealed that miR-27b is downregulated in most drug-resistant Ehrlich ascites tumor cells, suggesting this miRNA may be involved in drug resistance (68), even though no further results have been reported. miR-150 was found to have low expression in most primary ovarian tumors with significantly increased expression in omental lesions, and miR-150 increased the number of residual surviving cells by 2 - to 4 -fold when challenged with lethal cisplatin concentrations, indicating that the upregulation of miR-150 may stimulate survival and increase drug tolerance. Thus, miR-150 may be a critical regulator for the emergence of drug-resistant disease (69). miR-374a was found to be downregulated in head and neck squamous cell carcinoma cells exposed to cisplatin, resulting in the subsequent modulation of mRNA expression of several targets including DICER1, DDIT1 and DDIT4 which are involved in the apoptotic process. This indicates that miR-374a may mediate key pathways implicated in the response of cancer cells to chemotherapeutic drugs (70). miR-630 was found to arrest non-small cell lung cancer A549 


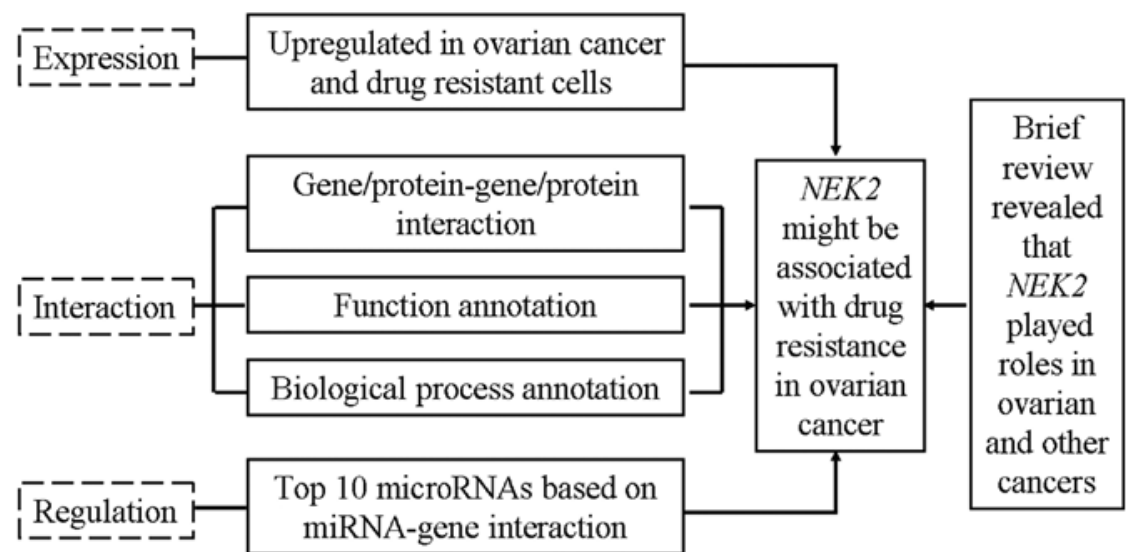

Figure 4. Overall procedure of the bioinformatic analysis of NEK2 associated with drug resistance in ovarian cancer. NEK2, NIMA (never in mitosis gene A)-related expressed kinase 2.

cells in the G0-G1 phase of the cell cycle, correlating with increased levels of the cell cycle inhibitor p27 as well as with reduced proliferation rates resulting in greatly diminished sensitivity of A549 cells to the late S-G2-M cell cycle arrest mediated by cisplatin. These results suggest that miR-630 may be a novel modulator of the cisplatin response in non-small cell lung cancer (71). In addition, miR-24-2 is capable of inducing apoptosis by modulating different apoptotic pathways and targeting BCL-2. Furthermore, cells overexpressing miR-24-2 are hypersensitive to DNA-damaging drugs such as cisplatin and undergo apoptotic cell death, suggesting that miR-24-2 may be involved in drug resistance in human cancer cells (MCF-7 and HeLa) (72). miR-128 is obviously expressed at a higher level in drug-resistant breast cancer samples than its expression in drug-sensitive samples. Following transfection with a precursor of miR-128 or antisense-miR-128 oligonucleotides, the chemosensitivity of MDA-MB-231 cell was upregulated (73), suggesting a direct association of miR-128 with drug resistance in cancer.

In regards to miR-216b and miR-486-5p, no study has indicated their associations with drug resistance in cancer, but they contribute to several biological processes during cancer development. It has been proven that downregulated expression of miR-216b is directly related to advanced clinical stage and lymph node metastasis. Both in vitro and in vivo assays revealed that miR-216b attenuates cell proliferation, invasion and tumor growth in nude mice (74). In addition, miR-216b, miR-186, miR-337-3p and miR-760 were found to cooperatively promote cellular senescence through the p53-p21 (Cip1/ WAF1) pathway in human colorectal cancer cells (75). Given that the overexpression of miR-486-5p is closely correlated with the downregulated expression of ARHGAP5 in lung tumor tissues, which considerably inhibits lung cancer cell migration and invasion, miR-486-5p may act as a tumor suppressor contributing to the progression and metastasis of non-small cell lung cancer (76). Actually, the biological processes including cell proliferation, invasion, growth and metastasis in which miR-216b and miR-486-5p are involved are closely related to the development of drug resistance in cancers $(49,77)$, and the annotation of biological processes also suggests that cell proliferation and growth are associated with drug resistance. All these results indicate that miR-216b and miR-486-5p may be associated with drug resistance in an indirectly way.

\section{Discussion}

Inferring the functional role of proteins/genes is a primary task in biology, for purposes ranging from general knowledge to drug discovery and diagnostic development (78). Protein/gene function prediction based on various genome-wide data is a potential, feasible and valuable strategy for gene function mining, and many large-scale networks of molecular interactions within the cell have made it possible to go beyond the one dimensional approach to study protein function in the context of a network (79). For example, on the basis of comprehensive bioinformatic analysis, Yin et al (77) reported that SPARCL1 and CCL21 are associated with drug resistance in ovarian cancer. Similarly, 15 TSGs associated with drug resistance in ovarian cancer were analyzed by comprehensive bioinformatics to overview the relationship of the 15 TSGs with drug resistance and to discover potential TSGs related with drug resistance (49).

Oncomine is a cancer microarray database and web-based data-mining platform aimed at facilitating the discovery from genome-wide expression analyses. Differential expression analyses comparing most major types of cancer with respective normal tissues are available for exploration. Data can be queried and visualized for a selected gene across all analyses (80). The Gene Expression Omnibus (GEO) at the National Center for Biotechnology Information (NCBI) has emerged as the leading fully public repository for gene expression data, predominantly gene expression data generated by DNA microarray technology (23). By 2006, GEO stored approximately a billion individual gene expression measurements, derived from over 100 organisms, submitted by over 1,500 laboratories, addressing a wide range of biological phenomena (81). Thus, the expression data of NEK2 in ovarian cancer tissues and drug-resistant cells retrieved from the two online databases are reliable.

GeneMANIA is a web-based database and a tool for the prediction of gene functions on the basis of 
multiple networks derived from different genomic or proteomic data/sources (24). Six organisms are currently supported (Arabidopsis thaliana, Caenorhabditis elegans, Drosophila melanogaster, Mus musculus, Homo sapiens and Saccharomyces cerevisiae), and hundreds of data sets have been collected from GEO, BioGRID, Pathway Commons and I2D, as well as organism-specific functional genomics data sets (82). Thus, it is fast enough to predict gene functions with great accuracy using this software. COREMINE Medical is a product of the PubGene Company designed to be used for searching information on health, medicine and biology (25). COREMINE Medical grew out of Pubgene online tool which is a gene/protein database and a web-based tool for literature mining. Pubgene carries out automated extraction of experimental and theoretical biomedical knowledge from publicly available gene and text databases to create a gene-to-gene co-citation network for millions of named human genes by automated analysis of titles and abstracts in over 10 million MEDLINE records (83). Therefore, the functions of NEK2 predicted by these two software programs were potentially accurate.

miRNAs are a class of small (22 bp) endogenous non-coding RNAs which regulate gene expression at the post-transcriptional and translational levels $(84,85)$. miRNA-mediated post-transcriptional gene regulation is considered as a significant regulator of many cellular processes including cell proliferation, apoptosis, differentiation, angiogenesis, invasion, metastasis and drug resistance, both physiological and pathological (86-88). miRNAs perform their functions through the regulation of their target genes, and it has been well established that miRNAs represent a class of genes with a great potential for use in diagnostics, prognosis and therapy (89). Therefore, we can predict the function of genes through the functions of the miRNAs targeting the gene. miRWalk is a comprehensive database on miRNAs, which collects predicted and validated miRNA binding sites on all known genes of the human, mouse and rat. More importantly, the miRWalk is a potential real-time database, in which the 'Predicted Target module' is updated every 6 months and the 'Validated Target module' is updated every month (26).

Taken together, based on the comprehensive bioinformatic analysis including microarray data interpretation, protein/gene-protein/gene interaction, annotation of biological processes and miRNA-mRNA interaction (Fig. 4), we revealed that the expression of NEK2 in drug-resistant ovarian cancer cells was elevated and that NEK2 directly/indirectly interacts with 20 proteins/genes which all contribute to drug resistance in ovarian and other cancers (Fig. 2). Further analysis based on the annotation of biological processes suggested that NEK2 was closely related to 14 biological processes which are involved in ovarian cancer and drug resistance (Fig. 3). Moreover, 7 of 10 miRNAs targeting NEK2 are involved in drug resistance in ovarian and other cancers (Table II). Given that NEK2 interacts with numerous proteins/genes, miRNAs and biological processes which were all found to contribute to drug resistance in ovarian and other cancers, upregulation of NEK2 in ovarian cancer and drug-resistant cancer cells may also contribute to drug-resistance.

Annotation of biological processes revealed that among the 14 biological processes annotated for NEK2, 6 were cell cycle- related (Fig. 3), and the molecular function annotation-based protein/gene-protein/gene interaction obtained similar results. These results suggest that NEK2 may participate in the regulation of drug resistance mainly through regulation of the cell cycle. In addition, microtubule-related function was another major function of NEK2 with its interacting proteins in accordance with protein/gene-protein/gene interaction (Table I), which implicated that NEK2 may also exert its drug resistant functions via microtubules. Actually, NEK2 has been reported to play important roles in both mitosis and meiosis (90) and contributes to the establishment of the microtubule-based mitotic spindle (27).

Collectively, for the first time, we report that the overexpression of NEK2 in ovarian cancers/drug-resistant cells may contribute to drug resistance through regulation of the cell cycle and microtubules. The present study provides important information for further investigation of the drug resistant-related functions of NEK2 in ovarian cancer.

\section{Acknowledgements}

The present study was supported by the National Natural Science Foundation of China (grant no. 81302283) and the Youth Science Foundation of Guangxi Medical University (no. GXMUYSF201205).

\section{References}

1. Landis SH, Murray T, Bolden S and Wingo PA: Cancer statistics, 1999. CA Cancer J Clin 49: 8-31, 1999.

2. Siegel R, Naishadham D and Jemal A: Cancer statistics, 2012. CA Cancer J Clin 62: 10-29, 2012.

3. Vaughan S, Coward JI, Bast RC Jr, Berchuck A, Berek JS, BrentonJD,Coukos G,Crum CC,DrapkinR,EtemadmoghadamD, Friedlander M, Gabra H, Kaye SB, Lord CJ, Lengyel E, Levine DA, McNeish IA, Menon U, Mills GB, Nephew KP, Oza AM, Sood AK, Stronach EA, Walczak H, Bowtell DD and Balkwill FR: Rethinking ovarian cancer: recommendations for improving outcomes. Nat Rev Cancer 11: 719-725, 2011.

4. Jemal A, Siegel R, Ward E, Hao Y, Xu J, Murray T and Thun MJ: Cancer statistics, 2008. CA Cancer J Clin 58: 71-96, 2008.

5. Cannistra SA: Cancer of the ovary. N Engl J Med 351: 2519-2529, 2004.

6. Gottesman MM: Mechanisms of cancer drug resistance. Annu Rev Med 53: 615-627, 2002.

7. Johnson SW, Ozols RF and Hamilton TC: Mechanisms of drug resistance in ovarian cancer. Cancer 71 (Suppl 2): S644-S649, 1993.

8. Sorrentino A, Liu CG, Addario A, Peschle C, Scambia G and Ferlini C: Role of microRNAs in drug-resistant ovarian cancer cells. Gynecol Oncol 111: 478-486, 2008.

9. Osborne C, Wilson P and Tripathy D: Oncogenes and tumor suppressor genes in breast cancer: potential diagnostic and therapeutic applications. Oncologist 9: 361-377, 2004.

10. Van Waardenburg RC, Prins J, Meijer C, Uges DR, De Vries EG and Mulder NH: Effects of c-myc oncogene modulation on drug resistance in human small cell lung carcinoma cell lines. Anticancer Res 16: 1963-1970, 1996.

11. Kumari R, Li H, Haudenschild DR, Fierro F, Carlson CS, Overn P, Gupta L, Gupta K, Nolta J, Yik JH and Di Cesare PE: The oncogene LRF is a survival factor in chondrosarcoma and contributes to tumor malignancy and drug resistance. Carcinogenesis 33: 2076-2083, 2012.

12. Barre B, Vigneron A, Perkins N, Roninson IB, Gamelin E and Coqueret O: The STAT3 oncogene as a predictive marker of drug resistance. Trends Mol Med 13: 4-11, 2007.

13. Zhao M, Sun J and Zhao Z: Distinct and competitive regulatory patterns of tumor suppressor genes and oncogenes in ovarian cancer. PLoS One 7: e44175, 2012. 
14. Ratner ES, Keane FK, Lindner R, Tassi RA, Paranjape T, Glasgow M, Nallur S, Deng Y, Lu L, Steele L, Sand S, Muller RU, Bignotti E, Bellone S, Boeke M, Yao X, Pecorelli S, Ravaggi A, Katsaros D, Zelterman D, Cristea MC, Yu H, Rutherford TJ, Weitzel JN, Neuhausen SL, Schwartz PE, Slack FJ, Santin AD and Weidhaas JB: A KRAS variant is a biomarker of poor outcome, platinum chemotherapy resistance and a potential target for therapy in ovarian cancer. Oncogene 31: 4559-4566, 2012.

15. Wu L, Wu A and Jiang K: Effect of antisense c-erbB2 on biologic behaviour and chemotherapeutic drug sensitivity in human ovarian cancer cells. Zhonghua Fu Chan Ke Za Zhi 31: 169-172, 1996 (In Chinese).

16. Fu S, Hennessy BT, Ng CS, Ju Z, Coombes KR, Wolf JK, Sood AK, Levenback CF, Coleman RL, Kavanagh JJ, Gershenson DM, Markman M, Dice K, Howard A, Li J, Li Y, Stemke-Hale K, Dyer M, Atkinson E, Jackson E, Kundra V, Kurzrock R, Bast RC Jr and Mills GB: Perifosine plus docetaxel in patients with platinum and taxane resistant or refractory high-grade epithelial ovarian cancer. Gynecol Oncol 126: 47-53, 2012

17. Lee S, Choi EJ, Jin C and Kim DH: Activation of PI3K/Akt pathway by PTEN reduction and PIK3CA mRNA amplification contributes to cisplatin resistance in an ovarian cancer cell line. Gynecol Oncol 97: 26-34, 2005.

18. Stronach EA, Alfraidi A, Rama N, Datler C, Studd JB, Agarwal R, Guney TG, Gourley C, Hennessy BT, Mills GB, Mai A, Brown R, Dina R and Gabra H: HDAC4-regulated STAT1 activation mediates platinum resistance in ovarian cancer. Cancer Res 71: 4412-4422, 2011

19. Fry AM, Schultz SJ, Bartek J and Nigg EA: Substrate specificity and cell cycle regulation of the Nek2 protein kinase, a potential human homolog of the mitotic regulator NIMA of Aspergillus nidulans. J Biol Chem 270: 12899-12905, 1995.

20. Lee J and Gollahon L: Nek2-targeted ASO or siRNA pretreatment enhances anticancer drug sensitivity in triplenegative breas cancer cells. Int J Oncol 42: 839-847, 2013.

21. Zhou W, Yang Y, Xia J, Wang H, Salama ME, Xiong W, Xu H, Shetty S, Chen T, Zeng Z, Shi L, Zangari M, Miles R, Bearss D, Tricot $\mathrm{G}$ and Zhan F: NEK2 induces drug resistance mainly through activation of efflux drug pumps and is associated with poor prognosis in myeloma and other cancers. Cancer Cell 23: 48-62, 2013

22. Harrison C: Cancer: a target for drug resistance. Nat Rev Drug Discov 12: 190, 2013. doi:10.1038/nrd3966.

23. Barrett T and Edgar R: Mining microarray data at NCBI's Gene Expression Omnibus (GEO)*. Methods Mol Biol 338: 175-190, 2006.

24. Mostafavi S, Ray D, Warde-Farley D, Grouios C and Morris Q GeneMANIA: a real-time multiple association network integration algorithm for predicting gene function. Genome Biol 9 (Suppl 1): S4, 2008

25. de Leeuw N, Dijkhuizen T, Hehir-Kwa JY, Carter NP, Feuk L, Firth HV, Kuhn RM, Ledbetter DH, Martin CL, van Ravenswaaij-Arts CM, Scherer SW, Shams S, Van Vooren S, Sijmons R, Swertz M and Hastings R: Diagnostic interpretation of array data using public databases and internet sources. Hum Mutat: Feb 14, 2012 (Epub ahead of print). doi: 10.1002/humu.22049.

26. Dweep H, Sticht C, Pandey P and Gretz N: miRWalk - database: prediction of possible miRNA binding sites by 'walking' the genes of three genomes. J Biomed Inform 44: 839-847, 2011

27. Fry AM, O'Regan L, Sabir SR and Bayliss R: Cell cycle regulation by the NEK family of protein kinases. J Cell Sci 125 4423-4433, 2012.

28. Andersen JS, Wilkinson CJ, Mayor T, Mortensen P, Nigg EA and Mann M: Proteomic characterization of the human centrosome by protein correlation profiling. Nature 426: 570-574, 2003.

29. Fry AM, Meraldi P and Nigg EA: A centrosomal function for the human Nek2 protein kinase, a member of the NIMA family of cell cycle regulators. EMBO J 17: 470-481, 1998.

30. Hayward DG, Clarke RB, Faragher AJ, Pillai MR, Hagan IM and Fry AM: The centrosomal kinase Nek2 displays elevated levels of protein expression in human breast cancer. Cancer Res 64 7370-7376, 2004.

31. Cappello P, Blaser H, Gorrini C, Lin DC, Elia AJ, Wakeham A, Haider S, Boutros PC, Mason JM, Miller NA, Youngson B, Done SJ and Mak TW: Role of Nek2 on centrosome duplication and aneuploidy in breast cancer cells. Oncogene: May 27, 2013 (Epub ahead of print). doi: 10.1038/onc.2013.183

32. Wang S, Li W, Lv S, Wang Y, Liu Z, Zhang J, Liu T and Niu Y: Abnormal expression of Nek 2 and $\beta$-catenin in breast carcinoma: clinicopathological correlations. Histopathology 59: 631-642, 2011.
33. Wang H, Xie YT, Han JY, Ruan Y, Song AP, Zheng LY, Zhang WZ, Sajdik C, Li Y, Tian XX and Fang WG: Genetic polymorphisms in centrobin and Nek2 are associated with breast cancer susceptibility in a Chinese Han population. Breast Cancer Res Treat 136: 241-251, 2012

34. Liu Z, Wang Y, Wang S, Zhang J, Zhang F and Niu Y: Nek2C functions as a tumor promoter in human breast tumorigenesis. Int J Mol Med 30: 775-782, 2012.

35. Landi MT, Dracheva T, Rotunno M, Figueroa JD, Liu H, Dasgupta A, Mann FE, Fukuoka J, Hames M, Bergen AW, Murphy SE, Yang P, Pesatori AC, Consonni D, Bertazzi PA, Wacholder S, Shih JH, Caporaso NE and Jen J: Gene expression signature of cigarette smoking and its role in lung adenocarcinoma development and survival. PLoS One 3: e1651, 2008.

36. Koch M and Wiese M: Gene expression signatures of angiocidin and darapladib treatment connect to therapy options in cervical cancer. J Cancer Res Clin Oncol 139: 259-267, 2013.

37. Hawkins SM, Loomans HA, Wan YW, Ghosh-Choudhury T, Coffey D, Xiao W, Liu Z, Sangi-Haghpeykar H and Anderson ML: Expression and functional pathway analysis of nuclear receptor NR2F2 in ovarian cancer. J Clin Endocrinol Metab 98: E1152-E1162, 2013.

38. Suzuki K, Kokuryo T, Senga T, Yokoyama Y, Nagino M and Hamaguchi M: Novel combination treatment for colorectal cancer using Nek2 siRNA and cisplatin. Cancer Sci 101: $1163-1169,2010$

39. Gudmundsdottir K and Ashworth A: The roles of BRCA1 and BRCA2 and associated proteins in the maintenance of genomic stability. Oncogene 25: 5864-5874, 2006.

40. Zhang F, Fan Q, Ren K and Andreassen PR: PALB2 functionally connects the breast cancer susceptibility proteins BRCA1 and BRCA2. Mol Cancer Res 7: 1110-1118, 2009.

41. Zhao D, Zhang W, Li XG, Wang XB, Li M, Li YF, Tian HM, Song PP, Liu J, Chang QY and Wu LY: The mRNA expression of BRCA1, ERCC1, TUBB3, PRR13 genes and their relationship with clinical chemosensitivity in primary epithelial ovarian cancer. Zhonghua Zhong Liu Za Zhi 34: 196-200, 2012 (In Chinese).

42. Lu L, Katsaros D, Wiley A, Rigault de la Longrais IA, Puopolo M and $\mathrm{Yu} \mathrm{H}$ : Expression of MDR1 in epithelial ovarian cancer and its association with disease progression. Oncol Res 16: 395-403, 2007.

43. Sakai W, Swisher EM, Jacquemont C, Chandramohan KV, Couch FJ, Langdon SP, Wurz K, Higgins J, Villegas E and Taniguchi T: Functional restoration of $B R C A 2$ protein by secondary BRCA2 mutations in BRCA2-mutated ovarian carcinoma. Cancer Res 69: 6381-6386, 2009.

44. Scully R, Chen J, Plug A, Xiao Y, Weaver D, Feunteun J, Ashley T and Livingston DM: Association of BRCA1 with Rad51 in mitotic and meiotic cells. Cell 88: 265-275, 1997.

45. Wu H, Cao Y, Weng D, Xing H, Song X, Zhou J, Xu G, Lu Y, Wang S and Ma D: Effect of tumor suppressor gene PTEN on the resistance to cisplatin in human ovarian cancer cell lines and related mechanisms. Cancer Lett 271: 260-271, 2008.

46. Yan X, Fraser M, Qiu Q and Tsang BK: Over-expression of PTEN sensitizes human ovarian cancer cells to cisplatin-induced apoptosis in a p53-dependent manner. Gynecol Oncol 102: 348-355, 2006

47. Zhang P, Gao W, Li H, Reed E and Chen F: Inducible degradation of checkpoint kinase 2 links to cisplatin-induced resistance in ovarian cancer cells. Biochem Biophys Res Commun 328: $567-572,2005$

48. Zhang X, Yashiro M, Qiu H, Nishii T, Matsuzaki T and Hirakawa K: Establishment and characterization of multidrug-resistant gastric cancer cell lines. Anticancer Res 30: 915-921, 2010.

49. Yin F, Liu X, Li D, Wang Q, Zhang W and Li L: Tumor suppressor genes associated with drug resistance in ovarian cancer (Review). Oncol Rep 30: 3-10, 2013

50. He G, Kuang J, Khokhar AR and Siddik ZH: The impact of S- and G2-checkpoint response on the fidelity of G1-arrest by cisplatin and its comparison to a non-cross-resistant platinum(IV) analog. Gynecol Oncol 122: 402-409, 2011.

51. Plumb JA, Strathdee G, Sludden J, Kaye SB and Brown R: Reversal of drug resistance in human tumor xenografts by 2'-deoxy-5-azacytidine-induced demethylation of the $h M L H 1$ gene promoter. Cancer Res 60: 6039-6044, 2000.

52. Strathdee G, MacKean MJ, Illand M and Brown R: A role for methylation of the $h M L H 1$ promoter in loss of hMLH1 expression and drug resistance in ovarian cancer. Oncogene 18: 2335-2341, 1999. 
53. Zhang X, Wang X, Song X, Liu C, Shi Y, Wang Y, Afonja O, Ma C, Chen YH and Zhang L: Programmed cell death 4 enhances chemosensitivity of ovarian cancer cells by activating death receptor pathway in vitro and in vivo. Cancer Sci 101: 2163-2170, 2010.

54. Reles A, Wen WH, Schmider A, Gee C, Runnebaum IB, Kilian U, Jones LA, El-Naggar A, Minguillon C, Schönborn I, Reich O, Kreienberg R, Lichtenegger W and Press MF: Correlation of p53 mutations with resistance to platinum-based chemotherapy and shortened survival in ovarian cancer. Clin Cancer Res 7: 2984-2997, 2001.

55. Al-Bahlani S, Fraser M, Wong AY, Sayan BS, Bergeron R, Melino G and Tsang BK: P73 regulates cisplatin-induced apoptosis in ovarian cancer cells via a calcium/calpain-dependent mechanism. Oncogene 30: 4219-4230, 2011.

56. Liu YY, Li L, Li DR, Zhang W and Wang Q: Suppression of WWOX gene by RNA interference reverses platinum resistance acquired in SKOV3/SB cells. Zhonghua Fu Chan Ke Za Zhi 43 854-858, 2008 (In Chinese).

57. Takai $\mathrm{N}$ and Narahara $\mathrm{H}$ : Histone deacetylase inhibitor therapy in epithelial ovarian cancer. J Oncol 2010: 458431, 2010.

58. Auner V, Sehouli J, Oskay-Oezcelik G, Horvat R, Speiser P and Zeillinger R: ABC transporter gene expression in benign and malignant ovarian tissue. Gynecol Oncol 117: 198-201, 2010.

59. Fink D, Nebel S, Aebi S, Nehme A and Howell S: Loss of DNA mismatch repair due to knockout of MSH2 or PMS2 results in resistance to cisplatin and carboplatin. Int J Oncol 11: 539-542, 1997.

60. Shah MA and Schwartz GK: Cell cycle-mediated drug resistance: an emerging concept in cancer therapy. Clin Cancer Res 7 : 2168-2181, 2001.

61. Li M, Balch C, Montgomery JS, Jeong M, Chung JH, Yan P, Huang TH, Kim S and Nephew KP: Integrated analysis of DNA methylation and gene expression reveals specific signaling pathways associated with platinum resistance in ovarian cancer. BMC Med Genomics 2: 34, 2009.

62. Gregory-Bass RC, Olatinwo M, Xu W, Matthews R, Stiles JK, Thomas K, Liu D, Tsang B and Thompson WE: Prohibitin silencing reverses stabilization of mitochondrial integrity and chemoresistance in ovarian cancer cells by increasing their sensitivity to apoptosis. Int J Cancer 122: 1923-1930, 2008.

63. Koshkin V and Krylov SN: Correlation between multi-drug resistance-associated membrane transport in clonal cancer cells and the cell cycle phase. PLoS One 7: e41368, 2012.

64. Fojo T and Menefee M: Mechanisms of multidrug resistance: the potential role of microtubule-stabilizing agents. Ann Oncol 18 (Suppl 5): v3-v8, 2007.

65. Kassler S, Donninger H, Birrer MJ and Clark GJ: RASSF1A and the taxol response in ovarian cancer. Mol Biol Int 2012: 263267, 2012.

66. Pellicciotta I, Yang CP, Venditti CA, Goldberg GL and Shahabi S: Response to microtubule-interacting agents in primary epithelial ovarian cancer cells. Cancer Cell Int 13: 33, 2013.

67. Li Z, Hu S, Wang J, Cai J, Xiao L, Yu L and Wang Z: MiR-27a modulates MDR1/P-glycoprotein expression by targeting HIPK2 in human ovarian cancer cells. Gynecol Oncol 119: 125-130, 2010.

68. Husted S, Søkilde R, Rask L, Cirera S, Busk PK, Eriksen J and Litman T: MicroRNA expression profiles associated with development of drug resistance in Ehrlich ascites tumor cells. Mol Pharm 8: 2055-2062, 2011.

69. Vang S, Wu HT, Fischer A, Miller DH, MacLaughlan S, Douglass E, Steinhoff M, Collins C, Smith PJ, Brard L and Brodsky AS: Identification of ovarian cancer metastatic miRNAs. PLoS One 8: e58226, 2013.

70. Huang Y, Chuang A, Hao H, Talbot C, Sen T, Trink B, Sidransky D and Ratovitski E: Phospho- $\Delta \mathrm{Np} 63 \alpha$ is a key regulator of the cisplatin-induced microRNAome in cancer cells. Cell Death Differ 18: 1220-1230, 2011

71. Galluzzi L, Morselli E, Vitale I, Kepp O, Senovilla L, Criollo A, Servant N, Paccard C, Hupé P, Robert T, Ripoche H, Lazar V, Harel-Bellan A, Dessen P, Barillot E and Kroemer G: miR-181a and miR-630 regulate cisplatin-induced cancer cell death. Cancer Res 70: 1793-1803, 2010.
72. Srivastava N, Manvati S, Srivastava A, Pal R, Kalaiarasan P, Chattopadhyay S, Gochhait S, Dua R and Bamezai RN: miR-24-2 controls $H 2 A F X$ expression regardless of gene copy number alteration and induces apoptosis by targeting antiapoptotic gene $B C L-2$ : a potential for therapeutic intervention. Breast Cancer Res 13: R39, 2011

73. Ji S, Shao G, Lv X, Liu Y, Fan Y, Wu A and Hu H: Downregulation of miRNA-128 sensitises breast cancer cell to chemodrugs by targeting Bax. Cell Biol Int 37: 653-658, 2013.

74. Deng M, Tang H, Zhou Y, Zhou M, Xiong W, Zheng Y, Ye Q, Zeng X, Liao Q, Guo X, Li X, Ma J and Li G: miR-216b suppresses tumor growth and invasion by targeting KRAS in nasopharyngeal carcinoma. J Cell Sci 124: 2997-3005, 2011.

75. Kim SY, Lee YH and Bae YS: MiR-186, miR-216b, miR-337-3p, and miR-760 cooperatively induce cellular senescence by targeting $\alpha$ subunit of protein kinase CKII in human colorectal cancer cells. Biochem Biophys Res Commun 429: 173-179, 2012.

76. Wang J, Tian X, Han R, Zhang X, Wang X, Shen H, Xue L, Liu Y, Yan X, Shen J, Mannoor K, Deepak J, Donahue JM, Stass SA, Xing L and Jiang F: Downregulation of miR-486-5p contributes to tumor progression and metastasis by targeting protumorigenic ARHGAP5 in lung cancer. Oncogene: Mar 11, 2013 (Epub ahead of print). doi: 10.1038/onc.2013.42

77. Yin F, Liu X, Li D, Wang Q, Zhang W and Li L: Bioinformatic analysis of chemokine (C-C motif) ligand 21 and SPARC-like protein 1 revealing their associations with drug resistance in ovarian cancer. Int J Oncol 42: 1305-1316, 2013.

78. Jiang X, Gold D and Kolaczyk ED: Network-based auto-probit modeling for protein function prediction. Biometrics 67: 958-966, 2011.

79. Sharan R, Ulitsky I and Shamir R: Network-based prediction of protein function. Mol Syst Biol 3: 88, 2007.

80. Rhodes DR, Yu J, Shanker K, Deshpande N, Varambally R, Ghosh D, Barrette T, Pandey A and Chinnaiyan AM: ONCOMINE: a cancer microarray database and integrated datamining platform. Neoplasia 6: 1-6, 2004.

81. Barrett T and Edgar R: Gene expression Omnibus: microarray data storage, submission, retrieval, and analysis. Methods Enzymol 411: 352-369, 2006.

82. Warde-Farley D, Donaldson SL, Comes O, Zuberi K, Badrawi R, Chao P, Franz M, Grouios C, Kazi F, Lopes CT, Maitland A, Mostafavi S, Montojo J, Shao Q, Wright G, Bader GD and Morris Q: The GeneMANIA prediction server: biological network integration for gene prioritization and predicting gene function. Nucleic Acids Res 38: W214-W220, 2010.

83. Jenssen TK, Laegreid A, Komorowski J and Hovig E: A literature network of human genes for high-throughput analysis of gene expression. Nat Genet 28: 21-28, 2001.

84. Behm-Ansmant I, Rehwinkel J and Izaurralde E: MicroRNAs silence gene expression by repressing protein expression and/or by promoting mRNA decay. Cold Spring Harb Symp Quant Biol 71: 523-530, 2006.

85. Bartel DP: MicroRNAs: genomics, biogenesis, mechanism, and function. Cell 116: 281-297, 2004.

86. Kloosterman WP and Plasterk RH: The diverse functions of microRNAs in animal development and disease. Dev Cell 11: 441-450, 2006

87. Croce CM and Calin GA: miRNAs, cancer, and stem cell division. Cell 122: 6-7, 2005.

88. Yi B, Piazza GA, Su X and Xi Y: MicroRNA and cancer chemoprevention. Cancer Prev Res 6: 401-409, 2013.

89. Tili E, Michaille JJ, Gandhi V, Plunkett W, Sampath D and Calin GA: miRNAs and their potential for use against cancer and other diseases. Future Oncol 3: 521-537, 2007.

90. Tanaka K, Parvinen $M$ and Nigg EA: The in vivo expression pattern of mouse Nek2, a NIMA-related kinase, indicates a role in both mitosis and meiosis. Exp Cell Res 237: 264-274, 1997. 